\title{
Correction to: Multi-fluid modelling of bubbly channel flows with an adaptive multi-group population balance method
}

\author{
D. Papoulias' $(\varangle)$, A. Vichansky ${ }^{2}$, M. Tandon ${ }^{3}$ \\ 1. Siemens Industries Software Computational Dynamics Ltd., 200 Shepherds Bush Road, London, W6 7NL, UK \\ 2. Siemens Industries Software Computational Dynamics Ltd., Basil Hill Road, Didcot, OX11 7HJ, UK \\ 3. Siemens Industries Software (India) Pvt.Ltd., Global Business Park Mehrauli-Gurgaon Road, Gurgaon, 122002, India
}

(๐) The Author(s) 2021, Corrected Publication November 2021

Correction to:

D. Papoulias, A. Vichansky, M. Tandon

Multi-fluid modelling of bubbly channel flows with an adaptive multi-group population balance method

Experimental and Computational Multiphase Flow 2021, 3(3): 171-185

https://doi.org/10.1007/s42757-020-0084-5

The article "Multi-fluid modelling of bubbly channel flows with an adaptive multi-group population balance method" written by D. Papoulias, A. Vichansky, and M. Tandon, was originally published electronically on the publisher's internet portal (currently SpringerLink) on 21 October 2020 without open access. After publication in Volume 3, Issue 3, page 171-185, the author(s) decided to opt for Open Choice and to make the article an open access publication. Therefore, the copyright of the article has been changed to (0 The Author(s) 2021 and the article is forthwith distributed under the terms of the Creative Commons Attribution 4.0 International License (http://creativecommons.org/licenses/by/4.0/), which permits use, duplication, adaptation, distribution and reproduction in any medium or format, as long as you give appropriate credit to the original author(s) and the source, provide a link to the Creative Commons license, and indicate if changes were made.

The original article has been corrected and can be found at https://doi.org/10.1007/s42757-020-0084-5.

$\triangle$ dimitrios.papoulias@cd-adapco.com

(10) UNIIVRSIYY PRESA 